\title{
Reaction of thiocarboxanilide derivatives of 2-phenylimino-3-phenyl-4-thiazolidinone and 1,3-diphenyl-2-thioxo-4-imidazolone with hydrazonoyl halides and active chloromethylene compounds
}

\author{
Hamdi M. Hassaneen ${ }^{1^{*}}$, Omar A. Miqdad ${ }^{2}$, Nada M. Abunada $^{2}$, Ahmad A. Fares $^{1}$ \\ ${ }^{1}$ Chemistry, Faculty of Science, Cairo University, Cairo, Egypt; ${ }^{*}$ Corresponding Author: hamdihass@gmail.com \\ ${ }^{2}$ Department of Chemistry, Faculty of Applied Sciences, Al-Aqsa University, Gaza, Palestine; \\ nadanadannrs@yahoo.com; miqdadomar@hotmail.com
}

Received 7 January 2011; revised 10 February 2011; accepted 13 February 2011

\begin{abstract}
The potassium salts of thiocarboxanilide of 2phenylimino-3-phenyl-4-thiazolidinone and 1,3diphenyl-2-thioxo-4-imidazolone react with hydrazonoyl halides in $\mathrm{N}, \mathrm{N}$-dimethylformamide to afford the corresponding 1,3,4-thiadiazoline derivatives. 2-Phenylimino-3-phenyl-4-thiazolidinone reacts with active chloromethylene compounds in $\mathrm{N}, \mathrm{N}$-dimethylformamide to give the corresponding thiazolylidenethiazolidin-4-one derivatives. The new compounds were characterized using IR, ${ }^{1} \mathrm{H}$ NMR, ${ }^{13} \mathrm{C}$ NMR and mass spectra.
\end{abstract}

Keywords: Hydrazonoyl Halides; 1,3,4-Thiadiazoline; Thiazolidinone

\section{INTRODUCTION}

1,3,4-Thiadiazole and its derivatives possess an interesting biological activity probably conferred to them by the strong aromaticity of this ring system [1]. It is known that many of its derivatives have antibacterial [2], antimicrobial [3], antimycobacterial [4,5], antifungal [6,7], antidepressant [8], anti-inflammatory [9], analgesic[10] activities and cardiotonic [11] action being notable. Besides, the thiazoline ring is associated with a variety of pharmacological actions, including antimicrobial [12], anti-inflammatory [13], anti-tumor [14], and antioxidant [15] actions. Moreover, imidazolinone derivatives constitute an important class of therapeutic activities such as anticonvulsant [16], potent central nervous system (CNS) depressant [17], and acting on $\alpha$-adrenergic and/or imidazoline receptors [18]. Recently, some new imidazolinone derivatives have been reported as antimicrobial $[19,20]$, histamine $\mathrm{H}_{3}$-receptor antagonist [21] and
L-DOPA prodrugs in the treatment of Parkinson's disease [22]. Some workers have recognized 5-imidazolone as having anticancer activity [20]. Prompted by these findings and due to our interest in the synthesis of new heterocyclic compounds with potential biological activities [23-26] and in continuation of our work on the synthesis of hetaryl-ylidene derivatives [27-29], we report herein the synthesis of some new 1,3,4-thiadiazoline(thiazoline)-2-hetarylylidene derivatives that might be of pharmacological importance.

\section{EXPERIMENTAL}

The melting points were determined on an electrothermal melting point apparatus and are uncorrected. IR spectra were recorded in $\mathrm{KBr}$ discs on a Pye Unicam SP 3300 and a Shimadzu FT-IR 8101 PC infrared spectrophotometers. The NMR spectra were recorded on a Varian Mercury VX-300 MHz NMR spectrometer in DMSO- $\mathrm{d}_{6}$ solutions using TMS as an internal reference. ${ }^{1} \mathrm{H}$ NMR spectra were run at $300 \mathrm{MHz}$ and ${ }^{13} \mathrm{C}$ NMR spectra were run at $75.46 \mathrm{MHz}$ in dimethylsulfoxide $\left(\right.$ DMSO- $\left.\mathrm{d}_{6}\right)$. Electron impact mass spectra were recorded on a Shimadzu GCMS-QP 1000 EX spectrometer at 70 $\mathrm{eV}$. Elemental analyses were carried out at the Micro Analytical Center at Cairo University, Giza, Egypt. 2-Phenylimino-3-phenyl-4-thiazolidinone 2 [30], 1,3diphenyl-2-thioxo- 4-imidazolone 13 [31] and hydrazonoyl halides 1a [32], 1b [33], 1c [34], 1d [35], 1e [36], 1f [37], 1g [38], 1h [39], 1i [40], 1j [41] and 1k [42] were prepared according to the reported literature methods.

Synthesis of 3,5-disubstituted 2-[2'-phenylimino-3'phenyl-4'-oxothiazolidin-5'-ylidene]-2,3-dihydro-1,3,4thiadiazole derivatives $7 \mathrm{a}-\mathrm{k}, 3,5$-disubstituted $2-[1,3$ 'diphenyl-2'-thioxo-4'-oxoimidazolidin-5'-ylidne]-2,3dihydro-1,3,4-thiadiazole derivatives $15 a-h$ and 2 - 
phenylimino-3-phenyl-5-[3'-phenyl-4',5'-disubstitutedthiazol-2'(3'H)ylidene]thiazolidin-4-one derivatives 19a-e: General method [29].

To a stirred suspension of potassium hydroxide $(0.23$ g, $5 \mathrm{mmol})$ in dimethylformamide $(20 \mathrm{ml})$ 2-phenylimino3 -phenyl-4-thiazolidinone 2 or $13(5 \mathrm{mmol})$ was added. The appropriate arylisothiocyanate $(5 \mathrm{mmol})$ was added to the resulting solution and the reaction mixture was stirred for $30 \mathrm{~min}$ at room temperature. A solution of the hydrazonoyl halide $1 \mathrm{a}-\mathrm{k}$ or active $\alpha$-chloromethylene compound 16a-e in dimethylformamide was then added to the reaction mixture and stirred for $24 \mathrm{~h}$ at room temperature, then treated with methanol $(10 \mathrm{ml})$. The solid formed was collected, washed with water and crystallized from a suitable solvent to give the respective $7 \mathrm{a}-\mathrm{k}$, $15 \mathrm{a}-\mathrm{h}$ and 19a-e.

3,5-Diphenyl-2-[2'-phenylimino-3'-phenyl-4'-oxoth iazolidin-5'-ylidene]-2,3-dihydro-1,3,4-thiadiazole 7a,

$\mathrm{mp} 270-1{ }^{\circ} \mathrm{C}$; $67 \%$ yield (dimethylformamide); IR (KBr) $v_{\max } / \mathrm{cm}^{-1} 1625.2(\mathrm{CO}) ;{ }^{1} \mathrm{H}$ NMR (DMSO-d $\left.\mathrm{d}_{6}\right) \delta$ 7.12-8.14 (m, Ar-H) ppm. ${ }^{13} \mathrm{C}$ NMR (DMSO-d $\left.{ }_{6}\right) \delta$ $120.37,120.75,123.92,124.15,125.12,125.30,126.31$, 128.11, 128.23, 129.32, 130.46, 131.44 (Ar-CH), 135.21, 137.77, 139.34, 139.64, 142.92, 155.26, 157.22, 159.97, 165.72 (Ar-C, C = C, C = N, C = O) ppm. MS m/z 504, 440, 282, 215, 179, 146, 91; Anal. Calcd. for $\mathrm{C}_{29} \mathrm{H}_{20} \mathrm{~N}_{4} \mathrm{OS}_{2}$ (504.62) C, 69.02; H, 3.99; N, 11.10; S, 12.71. Found: C, $69.10 ; \mathrm{H}, 4.00 ; \mathrm{N}, 11.00 ; \mathrm{S}, 12.69 \%$.

3-Phenyl-2-[2'-phenylimino-3'-phenyl-4'-oxothiazo lidin-5'-ylidene]-5-styryl-2,3-dihydro-1,3,4-thiadiazole 7b, mp $308-9^{\circ} \mathrm{C} ; 63 \%$ yield (dimethylformamide); IR (KBr) $v_{\max } / \mathrm{cm}^{-1} 1625.4(\mathrm{CO}) ;{ }^{1} \mathrm{H}$ NMR (DMSO-d $\left.{ }_{6}\right) \delta$ $6.62(\mathrm{~d}, \mathrm{~J}=15 \mathrm{~Hz}, 1 \mathrm{H}), 7.54(\mathrm{~d}, \mathrm{~J}=15 \mathrm{~Hz}, 1 \mathrm{H})$, 6.92-7.93 (m, 20H, Ar-H) ppm. ${ }^{13} \mathrm{C}$ NMR (DMSO-d $\left.\mathrm{d}_{6}\right) \delta$ $120.42,120.65,123.91,124.21,125.18,126.42,126.82$, $128.09,128.11,128.22,128.26,128.80,129.41,135.21$ $(\mathrm{Ar}-\mathrm{CH}, \mathrm{CH}=\mathrm{CH}), 137.81,138.94,139.66,142.09$, $142.87,154.72,155.24,159.83,165.70(\mathrm{Ar}-\mathrm{C}, \mathrm{C}=\mathrm{C}, \mathrm{C}=$ $\mathrm{N}, \mathrm{C}=\mathrm{O}) \mathrm{ppm} . \mathrm{MS} \mathrm{m} / \mathrm{z} 530,466,308,241,179,146$, 91; Anal. Calcd. for $\mathrm{C}_{31} \mathrm{H}_{22} \mathrm{~N}_{4} \mathrm{OS}_{2}(530.66) \mathrm{C}, 70.16 ; \mathrm{H}$, 4.18 ; N, 10.56; S, 12.08. Found: C, 70.20; H, 4.20; N, $10.52 ; \mathrm{S}, 12.10 \%$.

3-(4-Nitrophenyl)-2-[2'-phenylimino-3'-phenyl-4'oxothiazolidin-5'-ylidene]-5-(2-thienyl)-2,3-dihydro-1,3, 4-thiadiazole $7 \mathrm{c}, \mathrm{mp} 282-3^{\circ} \mathrm{C} ; 60 \%$ yield (ethanol); IR (KBr) $v_{\max } / \mathrm{cm}^{-1} 1629.1(\mathrm{CO}) ;{ }^{1} \mathrm{H}$ NMR $\left(\mathrm{DMSO}^{\left.-\mathrm{d}_{6}\right)} \delta\right.$ 6.84-8.17 (m, Ar-H) ppm. ${ }^{13} \mathrm{C}$ NMR (DMSO-d $\left.\mathrm{d}_{6}\right) \delta$ $119.02,120.36,123.71,123.88,125.26,125.92,128.19$, 128.26, 129.31, 134.71, 135.31, 136.72 (Ar-CH), 137.58, $139.58,142.87,143.21,144.52,155.23,157.26,159.74$, $165.68($ Ar-C, $\mathrm{C}=\mathrm{C}, \mathrm{C}=\mathrm{N}, \mathrm{C}=\mathrm{O}) \mathrm{ppm}$. MS m/z 555, 491, 332, 265, 224, 191, 136, 83, 51; Anal. Calcd. for $\mathrm{C}_{27} \mathrm{H}_{17} \mathrm{~N}_{5} \mathrm{O}_{3} \mathrm{~S}_{3}(555.65) \mathrm{C}, 58.36 ; \mathrm{H}, 3.08 ; \mathrm{N}, 12.60 ; \mathrm{S}$,
17.31. Found: C, 58.40; H, 3.10; N, 12.55; S, 17.23\%.

5-(2-Furyl)-3-(4-nitrophenyl)-2-[2'-phenylimino-3'phenyl-4'-oxothiazolidin-5'-ylidene]-2,3-dihydro-1,3, 4-thiadiazole $7 \mathrm{~d}, \mathrm{mp} 325-6^{\circ} \mathrm{C} ; 69 \%$ yield (dimethylformamide); IR (KBr) $v_{\max } / \mathrm{cm}^{-1} 1633.4(\mathrm{CO}) ;{ }^{1} \mathrm{H}$ NMR $\left(\right.$ DMSO-d $\left._{6}\right) \delta$ 6.59-8.31 (m, Ar-H) ppm. ${ }^{13} \mathrm{C}$ NMR $\left(\right.$ DMSO-d $\left._{6}\right) \delta 112.62,118.73,120.41,122.71,123.67$, $123.84,125.26,125.98,128.32,129.31,135.33,137.63$ (Ar-CH), 139.59, 143.28, 144.52, 148.23, 152.82, 155.32, 157.83, 159.78, $165.69(\mathrm{Ar}-\mathrm{C}, \mathrm{C}=\mathrm{C}, \mathrm{C}=\mathrm{N}, \mathrm{C}=\mathrm{O})$ ppm. MS m/z 539, 475, 316, 249, 224, 191, 136, 67, 51; Anal. Calcd. for $\mathrm{C}_{27} \mathrm{H}_{17} \mathrm{~N}_{5} \mathrm{O}_{4} \mathrm{~S}_{2}(539.58) \mathrm{C}, 60.10 ; \mathrm{H}$, 3.18 ; N, 12.98; S, 11.89. Found: C, 60.00; H, 3.20; N, $13.00 ; \mathrm{S}, 11.82 \%$.

3-(4-Nitrophenyl)-2-[2'-phenylimino-3'-phenyl-4'oxothiazolidin-5'-ylidene]-5-phenyl-2,3-dihydro-1,3, 4-thiadiazole 7e, $\mathrm{mp} 198-9^{\circ} \mathrm{C} ; 67 \%$ yield (ethanol); IR (KBr) $v_{\max } / \mathrm{cm}^{-1} 1630.3(\mathrm{CO}) ;{ }^{1} \mathrm{H}$ NMR (DMSO-d $\left.{ }_{6}\right) \delta$ 7.13-8.26 (m, Ar-H) ppm. ${ }^{13} \mathrm{C}$ NMR $\left(\right.$ DMSO-d $\left._{6}\right) \delta$ $118.72,120.34,123.63,123.86,125.27,125.30,125.84$, $128.19,129.33,130.44,131.24,135.40,137.64,137.75$, 139.61 (Ar-CH), 142.79, 144.54, 155.38, 157.31, 159.76, 165.66 (Ar-C, C = C, C = N, C = O) ppm. MS m/z 549, 485, 327, 260, 223, 191, 135, 77, 51; Anal. Calcd. for $\mathrm{C}_{29} \mathrm{H}_{19} \mathrm{~N}_{5} \mathrm{O}_{3} \mathrm{~S}_{2}$ (549.62) C, 63.37; H, 3.48; N, 12.74; S, 11.67. Found: C, 63.31; H, 3.44; N, 12.70; S, $11.61 \%$.

5-Acetyl-3-phenyl-2-[2'-phenylimino-3'-phenyl-4'oxothiazolidin-5'-ylidene]-2,3-dihydro-1,3,4-thiadiazole 7f, $\mathrm{mp} 250-2^{\circ} \mathrm{C}$; $68 \%$ yield (ethanol); IR (KBr) $v_{\max } / \mathrm{cm}^{-1}$ 1661.2 (CO acetyl), 1629.5 (CO thiazolinone); ${ }^{1} \mathrm{H}$ NMR $\left(\mathrm{DMSO}_{-} \mathrm{d}_{6}\right) \delta 2.35\left(\mathrm{~s}, 3 \mathrm{H}, \mathrm{CH}_{3}\right), 6.72-7.81(\mathrm{~m}, 15 \mathrm{H}$, Ar-H) ppm. ${ }^{13} \mathrm{C}$ NMR (DMSO-d 6 ) $\delta 24.76\left(\mathrm{CH}_{3}\right), 120.41$, $120.78,123.91,124.53,125.26,126.40,128.16,128.25$, 129.23 (Ar-CH), 135.34, 139.52, 139.65, 144.21, 155.31, 156.21, 159.02, 165.74, $188.54($ Ar-C, $\mathrm{C}=\mathrm{C}, \mathrm{C}=\mathrm{N}, \mathrm{C}=$ O) $\mathrm{ppm}$. MS m/z 470, 406, 248, 181, 178, 147, 91, 77, 51; Anal. Calcd. for $\mathrm{C}_{25} \mathrm{H}_{18} \mathrm{~N}_{4} \mathrm{O}_{2} \mathrm{~S}_{2}$ (470.08) C, 63.81; H, 3.86; N, 11.91; S, 13.63. Found: C, 63.74; H, 3.81; N, 11.87 ; $\mathrm{S}, 13.60 \%$.

Ethyl 3-phenyl-2-[2'-phenylimino-3'-phenyl-4'oxothiazolidin-5'-ylidene]-2,3-dihydro-1,3,4-thiadiazole-5-carboxylate $7 \mathrm{~g}, \mathrm{mp} 144-5^{\circ} \mathrm{C} ; 68 \%$ yield (ethanol); IR (KBr) $v_{\max } / \mathrm{cm}^{-1} 1745.1$ (CO ester), 1629.2 (CO thiazolinone); ${ }^{1} \mathrm{H}$ NMR (DMSO-d $\left.\mathrm{d}_{6}\right) \delta 1.41(\mathrm{t}, \mathrm{J}=7.2 \mathrm{~Hz}$, $\left.3 \mathrm{H}, \mathrm{CH}_{3}\right), 4.43\left(\mathrm{q}, \mathrm{J}=7.2 \mathrm{~Hz}, \mathrm{CH}_{2}\right), 6.72-7.41(\mathrm{~m}, 15 \mathrm{H}$, Ar-H) ppm. ${ }^{13} \mathrm{C}$ NMR (DMSO-d 6$) \delta 15.32\left(\mathrm{CH}_{3}\right), 64.17$ $\left(\mathrm{CH}_{2}\right), 119.97,120.39,123.82,123.96,125.31,125.39$, 128.21, 128.31, 129.14 (Ar-CH), 136.10, 139.36, 139.54, 144.62, 154.23, 154.67, 159.13, 165.81, 166.62 (Ar-C, $\mathrm{C}=\mathrm{C}, \mathrm{C}=\mathrm{N}, \mathrm{C}=\mathrm{O}) \mathrm{ppm} . \mathrm{MS} \mathrm{m} / \mathrm{z} 500,279,250,179$, 135, 103, 77, 51; Anal. Calcd. for $\mathrm{C}_{26} \mathrm{H}_{20} \mathrm{~N}_{4} \mathrm{O}_{3} \mathrm{~S}_{2}(500.59)$ C, 62.38; H, 4.03; N, 11.19; S, 12.81. Found: C, 62.34; $\mathrm{H}, 3.97 ; \mathrm{N}, 11.10 ; \mathrm{S}, 12.80 \%$. 
3-Phenyl-5-phenylaminocarbonyl-2-[2'-phenylimino3'-phenyl-4'-oxothiazolidin-5'-ylidene]-2,3-dihydro1,3,4-thiadiazole $7 \mathrm{~h}, \mathrm{mp} 307-8^{\circ} \mathrm{C} ; 70 \%$ yield (ethanol); IR (KBr) $v_{\max } / \mathrm{cm}^{-1} 1661.9$ (broad CO); ${ }^{1} \mathrm{H}$ NMR $\left(\right.$ DMSO-d $\left._{6}\right) \delta$ 7.34-8.41 (m, NH, Ar-H) ppm. ${ }^{13} \mathrm{C}$ NMR $\left(\right.$ DMSO-d $\left._{6}\right) \delta 120.36,121.12,122.38,122.79,124.12$, $124.54,124.97,125.61,128.18,128.31,128.75,128.91$ $(\mathrm{Ar}-\mathrm{CH}), \quad 135.41,139.42,139.67,140.66,146.68$, $153.14,157.87,159.18,165.32,165.45(\mathrm{Ar}-\mathrm{C}, \mathrm{C}=\mathrm{C}, \mathrm{C}$ $=\mathrm{N}, \mathrm{C}=\mathrm{O}) \mathrm{ppm} . \mathrm{MS} \mathrm{m} / \mathrm{z} 547,325,258,178,147,103$, 91, 77; Anal. Calcd. for $\mathrm{C}_{30} \mathrm{H}_{21} \mathrm{~N}_{5} \mathrm{O}_{2} \mathrm{~S}_{2}$ (547.65) C, 65.78; H, 3.87; N, 12.79; S, 11.71. Found: C, 65.81; H, 3.94; N, $12.83 ; \mathrm{S}, 11.66 \%$.

5-Benzoyl-3-phenyl-2-[2'-phenylimino-3'-phenyl-4'oxothiazolidin-5'-ylidene]-2,3-dihydro-1,3,4-thiadiazole 7i, $\mathrm{mp} 256-7^{\circ} \mathrm{C}$; $65 \%$ yield (ethanol); IR (KBr) $v_{\max } / \mathrm{cm}^{-1}$ 1674.3 (CO benzoyl), 1629.9 (CO thiazolinone); ${ }^{1} \mathrm{H}$ NMR (DMSO-d $\left.{ }_{6}\right) \delta$ 7.16-8.34 (m, Ar-H) ppm. ${ }^{13} \mathrm{C}$ NMR $\left(\right.$ DMSO-d $\left._{6}\right) \delta 120.32,120.39,123.98,124.54,125.21$, $126.39,128.24,128.27,129.12,129.19,129.81,133.47$ (Ar-CH), 135.41, 136.46, 139.43, 139.49, 143.92, 155.61, 156.21, 158.93, 165.80, 182.96 (Ar-C, C=C, C = $\mathrm{N}, \mathrm{C}=\mathrm{O}) \mathrm{ppm} . \mathrm{MS} \mathrm{m} / \mathrm{z} 532,310,243,178,163,147$, 103, 91, 77; Anal. Calcd. for $\mathrm{C}_{30} \mathrm{H}_{20} \mathrm{~N}_{4} \mathrm{O}_{2} \mathrm{~S}_{2}(532.63) \mathrm{C}$, 67.65; H, 3.78; N, 10.52; S, 12.04. Found: C, 67.70; H, $3.81 ; \mathrm{N}, 10.48 ; \mathrm{S}, 12.00 \%$.

3-Phenyl-2-[2'-phenylimino-3'-phenyl-4'-oxothiazolidin-5'-ylidene]-5-(2-thienoyl)-2,3-dihydro-1,3,4-thiadiazole $7 \mathbf{j}$, mp $122-3^{\circ} \mathrm{C}$; $66 \%$ yield (ethanol); IR (KBr) $v_{\max } / \mathrm{cm}^{-1}$ 1674.4 (CO thienoyl), 1625.6 (CO thiazolinone); ${ }^{1} \mathrm{H}$ NMR (DMSO-d $\left.{ }_{6}\right) \delta$ 6.92-8.34 (m, Ar-H) ppm. ${ }^{13} \mathrm{C}$ NMR (DMSO-d ${ }_{6}$ ) $\delta 119.84,120.40,124.31,124.45$, 125.30, 125.87, 128.19, 128.21, 128.32, 129.21, 135.11, 135.42 (Ar-CH), 137.19, 139.39, 139.48, 143.89, 144.12, 154.31, 155.91, 158.94, 165.28, 175.95 (Ar-C, C = C, C = $\mathrm{N}, \mathrm{C}=\mathrm{O}) \mathrm{ppm}$. MS m/z 538, 316, 249, 179, 91, 77; Anal. Calcd. for $\mathrm{C}_{28} \mathrm{H}_{18} \mathrm{~N}_{4} \mathrm{O}_{2} \mathrm{~S}_{3}(538.66) \mathrm{C}, 62.43 ; \mathrm{H}$, 3.37; N, 10.40; S, 17.86. Found: C, 62.40; H, 3.35; N, $10.36 ; \mathrm{S}, 17.90 \%$.

5-(2-Naphthoyl)-3-phenyl-2-[2'-phenylimino-3'phenyl-4'-oxothiazolidin-5'-ylidene]-2,3-dihydro-1,3, 4-thiadiazole $7 \mathrm{k}, \mathrm{mp} 118-9^{\circ} \mathrm{C}$; $68 \%$ yield (ethanol); IR (KBr) $v_{\max } / \mathrm{cm}^{-1} 1638.7$ (CO naphthoyl), 1629.9 (CO thiazolinone); ${ }^{1} \mathrm{H}$ NMR $\left(\right.$ DMSO-d $\left._{6}\right) \delta$ 7.15-8.39 (m, 21H, Ar-H), 8.85 (s, $1 \mathrm{H}$, naphthoyl $\alpha-\mathrm{H})$ ppm. ${ }^{13} \mathrm{C}$ NMR $\left(\right.$ DMSO-d $\left._{6}\right) \delta 119.68,120.32,123.68,124.16,125.23$, $125.49,126.40,126.58,127.76,127.84,128.10,128.18$, 129.23, 129.29, 129.87, 132.28 (Ar-CH), 132.51, 132.86, $135.50,135.61,139.44,142.26,144.18,155.29,155.63$, $159.13,165.82,183.60($ Ar-C, C $=\mathrm{C}, \mathrm{C}=\mathrm{N}, \mathrm{C}=\mathrm{O})$ ppm. MS m/z 582, 360, 293, 213, 181, 155, 103, 91, 77; Anal. Calcd. for $\mathrm{C}_{34} \mathrm{H}_{22} \mathrm{~N}_{4} \mathrm{O}_{2} \mathrm{~S}_{2}(582.69) \mathrm{C}, 70.08 ; \mathrm{H}$, 3.81 ; N, 9.62; S, 11.01. Found: C, 70.10; H, 3.80; N,
9.57; S, $11.00 \%$.

3,5-diphenyl-2-[1',3'-diphenyl-2'-thioxo-4'-oxoimidazolidin-5'-ylidne]-2,3-dihydro-1,3,4-thiadiazole $15 \mathrm{a}$, $\mathrm{mp} 136-8^{\circ} \mathrm{C}$; $63 \%$ yield (ethanol); IR $(\mathrm{KBr}) v_{\max } / \mathrm{cm}^{-1}$ 1670.1 (CO); ${ }^{1} \mathrm{H}$ NMR (DMSO-d 6 ) $\delta$ 7.12-8.19 (m, Ar-H) ppm. ${ }^{13} \mathrm{C}$ NMR (DMSO-d 6 ) $\delta 120.31,120.40,120.73$, $123.84,123.98,124.10,125.31,127.93,128.10,128.21$, 130.36, 131.43 (Ar-CH), 137.71, 138.92, 139.41, 139.52, 142.93, 156.17, 157.22, 165.74, 178.62 (Ar-C, C = C, C = $\mathrm{N}, \mathrm{C}=\mathrm{O}, \mathrm{C}=\mathrm{S}) \mathrm{ppm} . \mathrm{MS} \mathrm{m} / \mathrm{z} 504,341,274,238,206$, 135, 103, 91, 77; Anal. Calcd. for $\mathrm{C}_{29} \mathrm{H}_{20} \mathrm{~N}_{4} \mathrm{OS}_{2}$ (504.62) C, 69.02; H, 3.99; N, 11.10; S, 12.71. Found: C, 69.00; $\mathrm{H}, 4.00 ; \mathrm{N}, 11.00 ; \mathrm{S}, 12.65 \%$.

3-Phenyl-2-[1',3'-diphenyl-2'-thioxo-4'-oxoimidazolidin-5'-ylidne]-5-styryl-2,3-dihydro-1,3,4-thiadiazole 15b, mp $151-2^{\circ} \mathrm{C}$; $65 \%$ yield (ethanol); IR (KBr) $v_{\max } /$ $\mathrm{cm}^{-1} 1675.3(\mathrm{CO}) ;{ }^{1} \mathrm{H}$ NMR (DMSO-d $\left.\mathrm{d}_{6}\right) \delta 6.81(\mathrm{~d}, \mathrm{~J}=15$ $\mathrm{Hz}, 1 \mathrm{H}), 7.63(\mathrm{~d}, \mathrm{~J}=15 \mathrm{~Hz}, 1 \mathrm{H}), 7.26-7.83(\mathrm{~m}, 20 \mathrm{H}$, Ar-H) ppm. ${ }^{13} \mathrm{C}$ NMR (DMSO-d $\left.{ }_{6}\right) \delta 119.64,119.85$, $120.61,123.78,123.94,124.12,126.78,127.92,128.10$, $128.15,128.19,128.23,128.71,137.76(\mathrm{Ar}-\mathrm{CH}, \mathrm{CH}=$ $\mathrm{CH}), 138.96,139.12,140.12,142.01,143.26,154.34$, 155.31, 165.74, $178.51($ Ar-C, $\mathrm{C}=\mathrm{C}, \mathrm{C}=\mathrm{N}, \mathrm{C}=\mathrm{O}, \mathrm{C}=$ S) ppm. MS m/z 530, 367, 276, 206, 135, 103, 91, 77; Anal. Calcd. for $\mathrm{C}_{31} \mathrm{H}_{22} \mathrm{~N}_{4} \mathrm{OS}_{2}(530.66) \mathrm{C}, 70.16$; H4.18, ; N, 10.56; S, 12.08. Found: C, 70.10; H, 4.15; N, 10.50; S, $12.00 \%$.

3-(4-Nitrophenyl)-2-[1',3'-diphenyl-2'-thioxo-4'oxoimidazolidin-5'-ylidne]-5-(2-thienyl)-2,3-dihydro1,3,4-thiadiazole $15 \mathrm{c}, \mathrm{mp} 144-5^{\circ} \mathrm{C} ; 70 \%$ yield (ethanol); IR (KBr) $v_{\max } / \mathrm{cm}^{-1} 1670.9(\mathrm{CO}) ;{ }^{1} \mathrm{H}$ NMR $\left(\mathrm{DMSO}_{-} \mathrm{d}_{6}\right) \delta$ 6.85-8.16 (m, Ar-H) ppm. ${ }^{13} \mathrm{C}$ NMR (DMSO-d $\left.\mathrm{d}_{6}\right) \delta$ $119.67,120.03,120.16,123.76,123.92,124.18,127.73$, $128.13,128.21,134.80,136.64,137.61$ (Ar-CH), 139.54, 139.62, 143.14, 143.34, 144.32, 155.31, 157.32, 165.73, $178.43(\mathrm{Ar}-\mathrm{C}, \mathrm{C}=\mathrm{C}, \mathrm{C}=\mathrm{N}, \mathrm{C}=\mathrm{O}, \mathrm{C}=\mathrm{S}) \mathrm{ppm}$. MS $\mathrm{m} / \mathrm{z} 555,420,392,251,136,90,77,51$; Anal. Calcd. for $\mathrm{C}_{27} \mathrm{H}_{17} \mathrm{~N}_{5} \mathrm{O}_{3} \mathrm{~S}_{3}(555.65) \mathrm{C}, 58.36 ; \mathrm{H}, 3.08 ; \mathrm{N}, 12.60 ; \mathrm{S}$, 17.31. Found: C, 58.43; H, 3.05; N, 12.54; S, 17.27\%.

5-Acetyl-3-phenyl-2-[1',3'-diphenyl-2'-thioxo-4'oxoimidazolidin-5'-ylidne]-2,3-dihydro-1,3,4-thiadiazole $15 d, \mathrm{mp} 184-5^{\circ} \mathrm{C} ; 70 \%$ yield (ethanol); IR (KBr) $v_{\max } / \mathrm{cm}^{-1} 1734.2$ (CO acetyl), 1680.8 (CO imidazoline); ${ }^{1} \mathrm{H}$ NMR $\left(\right.$ DMSO-d $\left._{6}\right) \delta 2.42\left(\mathrm{~s}, 3 \mathrm{H}, \mathrm{CH}_{3}\right), 6.63-8.12(\mathrm{~m}$, $15 \mathrm{H}, \mathrm{Ar}-\mathrm{H}) \mathrm{ppm} .{ }^{13} \mathrm{C}$ NMR $\left(\mathrm{DMSO}-\mathrm{d}_{6}\right) \delta 24.84\left(\mathrm{CH}_{3}\right)$, 119.23, 120.26, 120.52, 124.18, 124.32, 124.51, 128.19 , 128.26, 128.34 (Ar-CH), 139.36, 139.48, 139.67, 144.32, 155.29, 156.51, 165.69, 179.63, 184.26 (Ar-C, C = C, C = $\mathrm{N}, \mathrm{C}=\mathrm{O}, \mathrm{C}=\mathrm{S}) \mathrm{ppm} . \mathrm{MS} \mathrm{m} / \mathrm{z} 470,406,307,240,135$, 103, 91, 77, 43; Anal. Calcd. for $\mathrm{C}_{25} \mathrm{H}_{18} \mathrm{~N}_{4} \mathrm{O}_{2} \mathrm{~S}_{2}$ (470.56) C, 63.81; H, 3.86; N, 11.91; S, 13.63. Found: C, 63.80; $\mathrm{H}, 3.82 ; \mathrm{N}, 11.90 ; \mathrm{S}, 13.60 \%$

Ethyl 3-Phenyl-2-[1',3'-diphenyl-2'-thioxo-4'-oxo- 
imidazolidin-5'-ylidne]-2,3-dihydro-1,3,4-thiadiazole5-carboxylate 15e, $\mathrm{mp} 221-3^{\circ} \mathrm{C}$; 70\% yield (ethanol); IR (KBr) $v_{\max } / \mathrm{cm}^{-1} 1750.8$ (CO ester), 1690.1 (CO imidazoline); ${ }^{1} \mathrm{H}$ NMR (DMSO-d 6 ) $\delta 1.41\left(\mathrm{t}, \mathrm{J}=7.2 \mathrm{~Hz}, \mathrm{CH}_{3}\right)$, $4.48\left(\mathrm{q}, \mathrm{J}=7.2 \mathrm{~Hz}, \mathrm{CH}_{2}\right), 6.83-7.54(\mathrm{~m}, 15 \mathrm{H}, \mathrm{Ar}-\mathrm{H}) \mathrm{ppm}$. ${ }^{13} \mathrm{C}$ NMR (DMSO-d $\left.\mathrm{d}_{6}\right) \delta 14.93\left(\mathrm{CH}_{3}\right), 64.31\left(\mathrm{CH}_{2}\right), 119.68$, 120.13, 120.42, 123.96, 124.16, 124.31, 128.22, 128.29, 128.34 (Ar-CH), 139.48, 139.54, 139.62, 144.61, 154.71, 155.21, 165.74, 166.52, $178.94($ Ar- $\mathrm{C}, \mathrm{C}=\mathrm{C}, \mathrm{C}=\mathrm{N}, \mathrm{C}=$ $\mathrm{O}, \mathrm{C}=\mathrm{S}) \mathrm{ppm}$. MS m/z 500, 337, 135, 103, 77, 51; Anal. Calcd. for $\mathrm{C}_{26} \mathrm{H}_{20} \mathrm{~N}_{4} \mathrm{O}_{3} \mathrm{~S}_{2}(500.59) \mathrm{C}, 62.38 ; \mathrm{H}, 4.03 ; \mathrm{N}$, 11.19 ; S, 12.81. Found: C, 62.34; H, 3.96; N, 11.20; S, $12.77 \%$.

3-Phenyl-2-[1',3'-diphenyl-2'-thioxo-4'-oxoimidazolidin-5'-ylidne]-5-phenylaminocarbonyl-2,3-dihydro1,3,4-thiadiazole 15f, $\mathrm{mp} 211-3^{\circ} \mathrm{C}$; $70 \%$ yield (ethanol); IR (KBr) $v_{\max } / \mathrm{cm}^{-1} 3387.3(\mathrm{NH}), 1696.1$ (CO imidazoline), 1671.4 (CO amide); ${ }^{1} \mathrm{H}$ NMR (DMSO-d $\left.{ }_{6}\right) \delta$ 7.10-8.42 (m, Ar-H, NH) ppm. ${ }^{13} \mathrm{C}$ NMR (DMSO-d $\left.{ }_{6}\right) \delta$ 119.84, 120.61, 121.31, 122.41, 122.80, 123.84, 124.16, $124.49,128.26,128.34,128.43,128.68$ (Ar-CH), 139.35, 139.62, 139.71, 140.37, 143.96, 155.43, 156.33, 165.46, 165.67, $179.36(\mathrm{Ar}-\mathrm{C}, \mathrm{C}=\mathrm{C}, \mathrm{C}=\mathrm{N}, \mathrm{C}=\mathrm{O}, \mathrm{C}=\mathrm{S}) \mathrm{ppm}$. MS m/z 547, 384, 178, 146, 120, 104, 91, 77; Anal. Calcd. for $\mathrm{C}_{30} \mathrm{H}_{21} \mathrm{~N}_{5} \mathrm{O}_{2} \mathrm{~S}_{2}(547.65) \mathrm{C}, 65.79 ; \mathrm{H}, 3.87 ; \mathrm{N}, 12.79 ; \mathrm{S}$, 11.71. Found: C, 65.74; H, 3.90; N, 12.81; S, 11.68\%.

5-Benzoyl-3-phenyl-2-[1',3'-diphenyl-2'-thioxo-4'oxoimidazolidin-5'-ylidne]-2,3-dihydro-1,3,4-thiadiazole 15g, mp $121-2^{\circ} \mathrm{C}$; $68 \%$ yield (ethanol); IR (KBr) $v_{\max } / \mathrm{cm}^{-1} 1650.9$ (broad CO); ${ }^{1} \mathrm{H}$ NMR (DMSO-d $\left.\mathrm{d}_{6}\right) \delta$ 7.19-8.46 (m, Ar-H) ppm. ${ }^{13} \mathrm{C}$ NMR $\left(\right.$ DMSO-d $\left._{6}\right) \delta$ $119.82,120.41,120.91,123.85,124.38,124.44,128.17$, 128.28, 128.32, 129.12, 129.75, 133.46 (Ar-CH), 136.51, 138.84, 139.26, 139.63, 143.84, 155.73, 156.31, 165.76, 178.76, $183.55(\mathrm{Ar}-\mathrm{C}, \mathrm{C}=\mathrm{C}, \mathrm{C}=\mathrm{N}, \mathrm{C}=\mathrm{O}, \mathrm{C}=\mathrm{S}) \mathrm{ppm}$. MS m/z 532, 369, 206, 135, 105, 91, 77; Anal. Calcd. for $\mathrm{C}_{30} \mathrm{H}_{20} \mathrm{~N}_{4} \mathrm{O}_{2} \mathrm{~S}_{2}(532.63) \mathrm{C}, 67.65 ; \mathrm{H}, 3.78 ; \mathrm{N}, 10.52 ; \mathrm{S}$, 12.04. Found: C, $67.70 ; \mathrm{H}, 3.81$; N, 10.49; S, $12.00 \%$.

3-Phenyl-2-[1',3'-diphenyl-2'-thioxo-4'-oxoimidazolidin-5'-ylidene]-5-(2-thienoyl)-2,3-dihydro-1,3,4-thiadiazole $15 \mathrm{~h}, \mathrm{mp} 167-8^{\circ} \mathrm{C}$; $70 \%$ yield (ethanol); IR $(\mathrm{KBr})$ $v_{\max } / \mathrm{cm}^{-1} 1650.8$ (broad CO); ${ }^{1} \mathrm{H}$ NMR (DMSO-d $\mathrm{d}_{6}$ ) $\delta$ 7.14-8.43 (m, Ar-H) ppm. ${ }^{13} \mathrm{C}$ NMR $\left(\right.$ DMSO-d $\left._{6}\right) \delta$ 119.67, 120.23, 120.91, 123.78, 124.13, 124.32, 127.93, 128.14, 128.19, 128.26, 135.12, 136.78 (Ar-CH), 139.34, 139.64, 140.12, 143.21, 144.13, 155.24, 156.32, 165.72, 174.86, $178.74($ Ar-C, $\mathrm{C}=\mathrm{C}, \mathrm{C}=\mathrm{N}, \mathrm{C}=\mathrm{O}, \mathrm{C}=\mathrm{S}) \mathrm{ppm}$. MS m/z 538, 375, 168, 137, 110, 91, 77; Anal. Calcd. for $\mathrm{C}_{28} \mathrm{H}_{18} \mathrm{~N}_{4} \mathrm{O}_{2} \mathrm{~S}_{3}$ (538.66) C, 62.43; H, 3.37; N, 10.40; S, 17.86. Found: C, 62.40 ; H, 3.34; N, 10.37; S, 17.82\%.

Ethyl 3-phenyl-4-methyl-2-[3'-phenyl-2'-phenylimino-4-oxothiazolidin-5'-ylidene]-2(3H) thiazolecarboxylate 19a, mp $294-5^{\circ} \mathrm{C} ; 61 \%$ yield (ethanol); IR
(KBr) $v_{\max } / \mathrm{cm}^{-1} 1696.5$ (CO ester), 1620.9 (CO thiazolinone); ${ }^{1} \mathrm{H}$ NMR (DMSO-d 6 ) $\delta 1.32\left(\mathrm{t}, \mathrm{J}=7.1 \mathrm{~Hz}, \mathrm{CH}_{3}\right.$ ), $2.23\left(\mathrm{~s}, 3 \mathrm{H}, \mathrm{CH}_{3}\right), 4.36\left(\mathrm{q}, \mathrm{J}=7.1 \mathrm{~Hz}, \mathrm{CH}_{2}\right), 6.73-7.54$ (m, $15 \mathrm{H}$, Ar-H) ppm. ${ }^{13} \mathrm{C}$ NMR (DMSO-d $\left.{ }_{6}\right) \delta 14.38$ $\left(\mathrm{CH}_{3}\right), 14.85\left(\mathrm{CH}_{3}\right), 63.92\left(\mathrm{CH}_{2}\right), 119.82,120.41,123.43$, 123.83, 125.24, 126.42, 127.93, 128.19, 129.25 (Ar-CH), 153.21, 136.40, 139.35, 140.13, 143.43, 155.42, 157.62, 159.46, 165.71, $166.55(\mathrm{Ar}-\mathrm{C}, \mathrm{C}=\mathrm{C}, \mathrm{C}=\mathrm{N}, \mathrm{C}=\mathrm{O}) \mathrm{ppm}$. MS m/z 513, 291, 203, 194, 90, 77, 73, 51; Anal. Calcd. for $\mathrm{C}_{28} \mathrm{H}_{23} \mathrm{~N}_{3} \mathrm{O}_{3} \mathrm{~S}_{2}(513.63) \mathrm{C}, 65.48 ; \mathrm{H}, 4.51 ; \mathrm{N}, 8.18 ; \mathrm{S}$, 12.49. Found: C, 65.51; H, 4.50; N, 8.20; S, $12.52 \%$.

2-Phenylimino-3-phenyl-5-[4'-methyl-3'-phenyl-5'phenylaminocarbonylthiazol-2'(3'H)ylidene] thiazolidin-4-one $19 b$, mp $323-5^{\circ} \mathrm{C} ; 70 \%$ yield (dimethylformamide); IR (KBr) $v_{\max } / \mathrm{cm}^{-1} 3340.2(\mathrm{NH}), 1660.6$ (broad CO); ${ }^{1} \mathrm{H}$ NMR (DMSO-d $\left.{ }_{6}\right) \delta 2.24\left(\mathrm{~s}, 3 \mathrm{H}, \mathrm{CH}_{3}\right)$, 6.74-7.53 (m, NH, 20H, Ar-H) ppm. ${ }^{13} \mathrm{C}$ NMR (DMSO$\left.\mathrm{d}_{6}\right) \delta 15.23\left(\mathrm{CH}_{3}\right), 119.82,120.42,122.10,122.63$, $124.10,124.23,125.34,125.92,128.13,128.25,128.62$, 129.26 (Ar-CH), 133.67, 136.21, 139.41, 140.02, 140.24, $143.13,155.42,156.78,160.34,165.81,166.98$ (Ar-C, $\mathrm{C}=\mathrm{C}, \mathrm{C}=\mathrm{N}, \mathrm{C}=\mathrm{O}) \mathrm{ppm}$. MS m/z 560, 338, 250, 194, 160, 120, 91, 77; Anal. Calcd. for $\mathrm{C}_{32} \mathrm{H}_{24} \mathrm{~N}_{4} \mathrm{O}_{2} \mathrm{~S}_{2}(560.68)$ C, 68.55; H, 4.31; N, 9.99; S, 11.44. Found: C, 68.61; H, $4.30 ; \mathrm{N}, 10.00 ; \mathrm{S}, 11.40 \%$.

2-Phenylimino-3-phenyl-5-[3',4'-diphenylthiazol-2' (3'H)ylidene]thiazolidin-4-one 19c, mp $278-9^{\circ} \mathrm{C}$; $65 \%$ yield (ethanol); IR ( $\mathrm{KBr}) v_{\max } / \mathrm{cm}^{-1} 1615.7$ (CO thiazolinone); ${ }^{1} \mathrm{H}$ NMR (DMSO-d $\left.\mathrm{d}_{6}\right) \delta$ 6.16-7.61 (m, 21H, Ar-H) ppm. ${ }^{13} \mathrm{C}$ NMR (DMSO-d ${ }_{6}$ ) $\delta 119.78,120.21,123.96$, 124.14, 125.23, 125.84, 126.30, 128.11, 128.31, 129.15, $130.12,130.93,132.62(\mathrm{Ar}-\mathrm{CH}, \mathrm{CH}=\mathrm{C}), 135.94,136.84$, 139.40, 140.02, 143.21, 143.63, 155.35, 159.68, 165.76 (Ar-C, C $=\mathrm{C}, \mathrm{C}=\mathrm{N}, \mathrm{C}=\mathrm{O}$ ) ppm. MS m/z 503, 281, 194, 121, 77; Anal. Calcd. for $\mathrm{C}_{30} \mathrm{H}_{21} \mathrm{~N}_{3} \mathrm{OS}_{2}(503.63) \mathrm{C}$, 71.54; H, 4.20; N, 8.34; S, 12.73. Found: C, 71.50; H, 4.16; N, $8.30 ; \mathrm{S}, 12.70 \%$.

2-Phenylimino-3-phenyl-5-[5'-benzoyl-3',4'-diphenylthiazol-2'(3'H)ylidene]thiazolidin-4-one $19 \mathrm{~d}, \mathrm{mp}$ $345-7^{\circ} \mathrm{C}$; $67 \%$ yield (dimethylformamide); IR ( $\mathrm{KBr}$ ) $v_{\max } / \mathrm{cm}^{-1} 1710.1$ (CO benzoyl), 1660.3 (CO thiazolinone); ${ }^{1} \mathrm{H}$ NMR (DMSO-d $\left.{ }_{6}\right) \delta$ 6.76-7.62 (m, 25H, Ar-H) ppm. ${ }^{13} \mathrm{C}$ NMR (DMSO-d $\left.\mathrm{d}_{6}\right) \delta 119.58,120.16,123.89,124.15$, $125.36,125.78,126.30,128.15,128.41,129.11,129.23$, 129.82, 130.26, 131.36, 133.39 (Ar-CH), 135.34, 135.74, 136.61, 136.84, 139.41, 139.91, 143.19, 155.24, 156.32, $159.69,166.42,184.18(\mathrm{Ar}-\mathrm{C}, \mathrm{C}=\mathrm{C}, \mathrm{C}=\mathrm{N}, \mathrm{C}=\mathrm{O}) \mathrm{ppm}$. MS m/z 607, 388, 387, 386, 385, 355, 308, 280, 265, 247, 194, 135, 121, 105, 77, 51; Anal. Calcd. for $\mathrm{C}_{37} \mathrm{H}_{25} \mathrm{~N}_{3} \mathrm{O}_{2} \mathrm{~S}_{2}$ (607.74) C, 73.12; H, 4.15; N, 6.91; S, 10.55. Found: C, 73.10; H, 4.10; N, 6.88; S, 10.51\%.

2-Phenylimino-3-phenyl-5-[4'-methyl-3'-phenylthiazol-2'(3'H)ylidene]thiazolidin-4-one $19 \mathrm{e}, \mathrm{mp} 220-2^{\circ} \mathrm{C}$; 
$69 \%$ yield (ethanol); IR (KBr) $v_{\max } / \mathrm{cm}^{-1} 1615.3(\mathrm{CO}$ thiazolinone); ${ }^{1} \mathrm{H}$ NMR (DMSO-d $\left.\mathrm{d}_{6}\right) \delta 1.85\left(\mathrm{~s}, 3 \mathrm{H}, \mathrm{CH}_{3}\right)$, 6.15-7.42 (m, 16H, Ar-H) ppm. ${ }^{13} \mathrm{C}$ NMR $\left(\right.$ DMSO-d $\left._{6}\right) \delta$ $15.42\left(\mathrm{CH}_{3}\right), 119.62,120.31,123.93,124.12,125.41$, $125.86,128.12,128.26,129.08,131.92(\mathrm{Ar}-\mathrm{CH}, \mathrm{CH}=$ C), 135.83, 139.34, 139.84, 143.21, 144.18, 155.34, 159.77, $165.77(\mathrm{Ar}-\mathrm{C}, \mathrm{C}=\mathrm{C}, \mathrm{C}=\mathrm{N}, \mathrm{C}=\mathrm{O}) \mathrm{ppm}$. MS $\mathrm{m} / \mathrm{z}$ 441, 219, 131, 121, 103, 91, 77; Anal. Calcd. for $\mathrm{C}_{25} \mathrm{H}_{19} \mathrm{~N}_{3} \mathrm{OS}_{2}$ (441.56) C, 68.00; H, 4.34; N, 9.52; S, 14.52. Found: C, $67.92 ; \mathrm{H}, 4.28 ; \mathrm{N}, 9.48 ; \mathrm{S}, 14.50 \%$.

\section{RESULTS AND DISCUSSION}

The intermediates $4 \mathrm{~A}\left(\mathrm{Ar}^{\prime}=\mathrm{ph}\right)$ and $4 \mathrm{~B}\left(\mathrm{Ar}^{\prime}=4-\right.$ $\mathrm{CH}_{3} \mathrm{C}_{6} \mathrm{H}_{4}$ ) were prepared by the reaction of 2-phenylimino-3-phenyl-4-thiazolidinone 2 with arylisothiocyanate $3 \mathrm{~A}, \mathrm{~B}$ in dimethylformamide in the presence of potassium hydroxide (Scheme 1). Treatment of 4A with hydrazonoyl halides la-e in dimethylformamide afforded, in each case, one isolable product as evidenced by TLC analysis of the crude products. Both mass and elemental
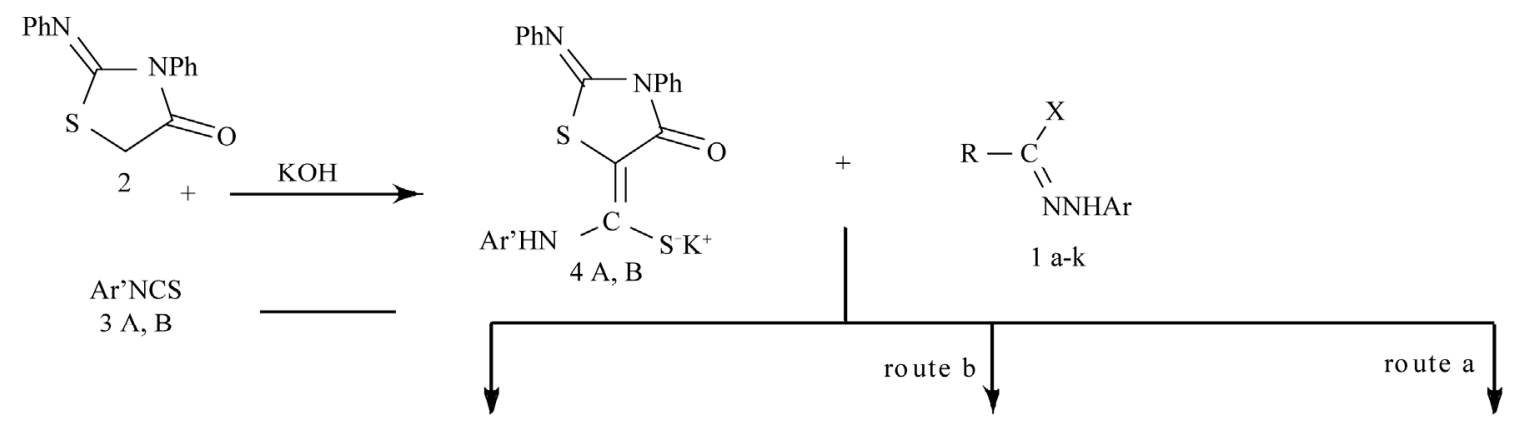

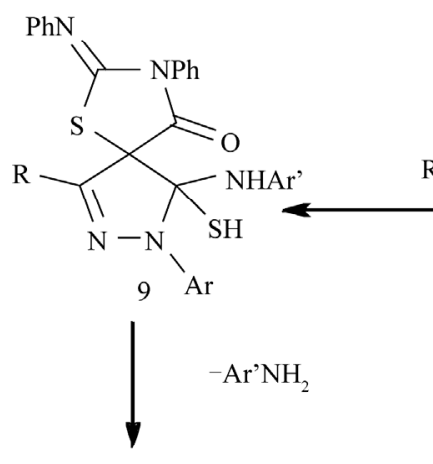<smiles>[R]C(=NN)C1(C(N)=O)SC(=N)NC1=O</smiles><smiles>[R]C1=NN([Al])C(=S)C12SC(=N)N[NH2+]C2=O</smiles>

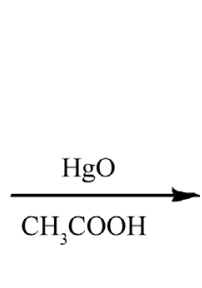

10

\begin{tabular}{|c|c|c|c|}
\hline 1,7 & $\mathrm{R} / \mathrm{Ar}$ & 1,7 & $\mathrm{R} / \mathrm{Ar}$ \\
\hline a & $\mathrm{C}_{6} \mathrm{H}_{5} / \mathrm{C}_{6} \mathrm{H}_{5}$ & $\mathrm{~g}$ & $\mathrm{COOC}_{2} \mathrm{H}_{5} / \mathrm{C}_{6} \mathrm{H}_{5}$ \\
\hline $\mathrm{b}$ & $\mathrm{C}_{6} \mathrm{H}_{5} \mathrm{CH}=\mathrm{CH} / \mathrm{C}_{6} \mathrm{H}_{5}$ & $\mathrm{~h}$ & $\mathrm{CONHPh} / \mathrm{C}_{6} \mathrm{H}_{5}$ \\
\hline $\mathrm{c}$ & 2-thienyl/4- $\mathrm{NO}_{2} \mathrm{C}_{6} \mathrm{H}_{4}$ & $\mathrm{i}$ & $\mathrm{COC}_{6} \mathrm{H}_{5} / \mathrm{C}_{6} \mathrm{H}_{5}$ \\
\hline d & 2-fury $/ 4-\mathrm{NO}_{2} \mathrm{C}_{6} \mathrm{H}_{4}$ & $\mathrm{j}$ & 2-thienyl/ $/ \mathrm{C}_{6} \mathrm{H}_{5}$ \\
\hline e & $\mathrm{C}_{6} \mathrm{H}_{5} / 4-\mathrm{NO}_{2} \mathrm{C}_{6} \mathrm{H}_{4}$ & k & 2-naphthoyl/ $\mathrm{C}_{6} \mathrm{H}_{5}$ \\
\hline f & $\mathrm{COCH}_{3} / \mathrm{C}_{6} \mathrm{H}_{5}$ & & \\
\hline
\end{tabular}

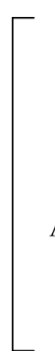<smiles>[R]C1=NN([Y19])C([C@@H]2[NH2+]NC(=N)S2)C1=O</smiles><smiles>[R]C1=NN([Y8])C(=C2SC(=N)[NH2+]C2=O)S1</smiles>

7

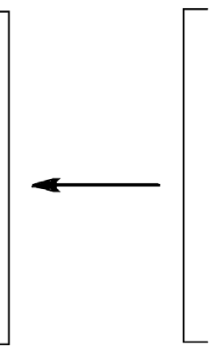<smiles>[R]C(=N)S/C([NH3+])=C1/SC(=N)NC1=O</smiles>

5<smiles>[R]C1=NN([Y9])C(=C2SC(=N)NC2=O)S1</smiles>

11

Scheme 1. 
analyses data of the products isolated are compatible with the two possible structures 7 and 10 (Scheme 1). However, the latter structure 10 was discarded as the reaction products were recovered unchanged after treatment with mercuric oxide in boiling acetic acid while the treatment is expected to convert 10 -if present- to 12 , the $\mathrm{C}=\mathrm{S}$ double bond is known to be more reactive dipolarophile than the $\mathrm{C}=\mathrm{C}$ double bond [43], and reaction of acyclic $\beta$-ketothioanilides with 1 has been reported to give 2-alkylidene derivatives [44]. Accordingly, the product isolated from reaction 1a with $4 \mathrm{~A}$ or $4 \mathrm{~B}$ is assigned structure 7 . This assignment was substantiated by the finding that reactions of $1 \mathrm{a}$ with either $4 \mathrm{~A}$ or $4 \mathrm{~B}$ yield one product which is the same in both cases indicating the elimination of an arylamine molecule during the reaction to give 7. To account for the formation of 7 it is suggested that the reaction starts with the formation of thiohydrazonate ester 5 followed by intramolecular cyclization to give 6 which in turn eliminated arylamine to give 7 .

Stereochemically, the isolated products can have either the 7 or 11 configurations. Molecular models indicate that structure 11 suffers severe steric interactions due to the close proximity of $\mathrm{N}$-aryl group and $\mathrm{C}=\mathrm{O}$ group. On this basis we suggest that the configuration of the products isolated is the less hindered structure 7 .

Similarly, treatment of 1,3-diphenyl-2-thioxo-4-imidazolinon-5-thiocarboxanilide 14A (prepared by the reaction of 1,3-diphenyl-2-thioxo-4-imidazolone 13 with phenylisothiocyanate $3 \mathrm{~A}$ in dimethylformamide in the presence of potassium hydroxide) with hydrazonoyl halides 1a-c,f-j afforded a single product in each case and was assigned structure 15 (Scheme 2). The structure of the latter products was established on the basis of its
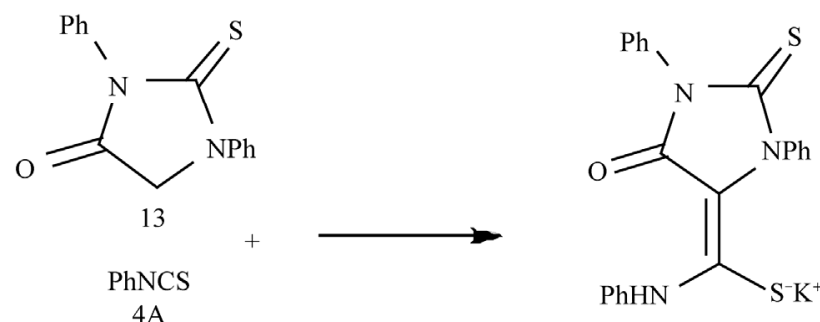

14

\begin{tabular}{ll}
15 & \multicolumn{1}{c}{$\mathrm{R} / \mathrm{Ar}$} \\
\hline $\mathrm{a}$ & $\mathrm{C}_{6} \mathrm{H}_{5} / \mathrm{C}_{6} \mathrm{H}_{5}$ \\
b & $\mathrm{C}_{6} \mathrm{H}_{5} \mathrm{CH}=\mathrm{CH} / \mathrm{C}_{6} \mathrm{H}_{5}$ \\
c & 2-thienyl $/ 4-\mathrm{NO}_{2} \mathrm{C}_{6} \mathrm{H}_{4}$ \\
d & $\mathrm{COCH}_{3} / \mathrm{C}_{6} \mathrm{H}_{5}$
\end{tabular}

$15 \mathrm{R} / \mathrm{Ar}$

e $\quad \mathrm{COOC}_{2} \mathrm{H}_{5} / \mathrm{C}_{6} \mathrm{H}_{5}$

f $\mathrm{CONHPh} / \mathrm{C}_{6} \mathrm{H}_{5}$

g $\quad \mathrm{COC}_{6} \mathrm{H}_{5} / \mathrm{C}_{6} \mathrm{H}_{5}$

h 2-thienyl $/ \mathrm{C}_{6} \mathrm{H}_{5}$

elemental analysis and spectroscopic data (Experimental). The IR spectrum of the isolated product $15 \mathrm{a}$, taken as example, revealed the appearance of ring carbonyl absorption band near $1670 \mathrm{~cm}^{-1}$ in addition, its mass spectrum revealed a peak corresponding to the molecular ion $\mathrm{m} / \mathrm{z} 504$.

In the course of the previous reaction it was found that the reaction proceeds via elimination of an arylamine to give the product. This finding promoted us to perform the reaction of $4 \mathrm{~A}$ with active $\alpha$-chloromethylene compounds 16a-e to investigate if such reaction will lead to thiazoline 19 and/or 1,3-oxathiol 20. Previous literature reports indicated that the reaction of active $\alpha$-chloromethylene compounds of simple ketones and nitriles with potassium salts of acyclic thioanilide gave the thiazoline derivatives [45], while with cyclic thioanilide gave 1,3-oxathiol derivatives [28].

Treatment of 4A with ethyl 2-chloro-3-oxobutanoate 16a in dimethylformamide afforded a single product as evidenced by TLC and ${ }^{1} \mathrm{H}-\mathrm{NMR}$ of the crude products (Scheme 3).

Both elemental and spectroscopic analyses data were found compatible with 2,3-dihydro-3-phenylthiazole derivatives structure 19 and not the 1,3-oxathiol-2-ylidene derivatives 20 we reported earlier [28]. Compound 4A reacted similarly with varieties of active $\alpha$-chloromethylene compounds $16 \mathrm{~b}-\mathrm{e}$ and gave the corresponding $19 \mathrm{~b}$-e respectively. The reaction pathway that seems to account for the formation of 19 from 5 and 16 is outlined in Scheme 3. It is proposed that the reaction involves nucleophilic substitution to give 17. Cyclization of the latter product leads to the formation of 18 which loses the elements of water to give 19 . These products can be assigned one of the two stereoisomeric structures (Z)-19

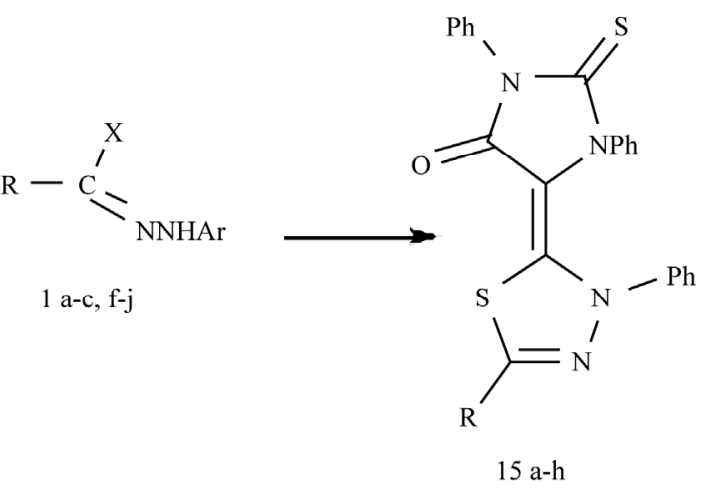

Scheme 2. 
<smiles>N=C1SC(=C(N)N)C(=O)N1c1ccccc1</smiles><smiles>[R]C(=O)C([R])Cl</smiles>

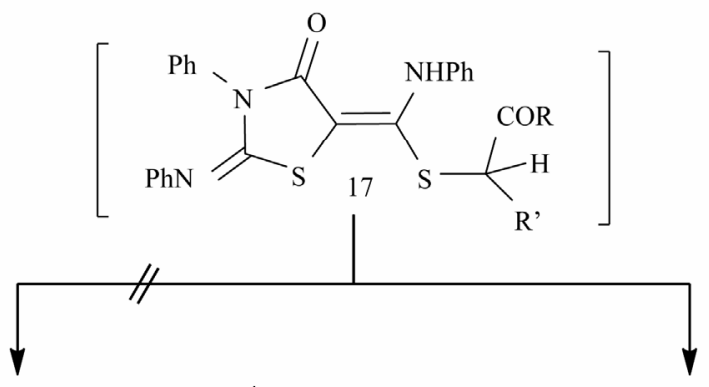<smiles>[R]C1=C([R])SC(=C2SC(=N)N(c3ccccc3)C2=O)O1</smiles>

\begin{tabular}{cl}
19 & \multicolumn{1}{c}{$\mathrm{R} / \mathrm{R}^{\prime}$} \\
\hline $\mathrm{a}$ & $\mathrm{CH}_{3} / \mathrm{COOC}_{2} \mathrm{H}_{5}$ \\
b & $\mathrm{CH}_{3} / \mathrm{CONHC}_{6} \mathrm{H}_{5}$ \\
c & $\mathrm{C}_{6} \mathrm{H}_{5} / \mathrm{H}$ \\
d & $\mathrm{C}_{6} \mathrm{H}_{5} / \mathrm{COC}_{6} \mathrm{H}_{5}$ \\
e & $\mathrm{CH}_{3} / \mathrm{H}$
\end{tabular}

Scheme 3 .

or (E)-19 (Scheme 3). The present data cannot distinguish between these two isomers, however. The elemental analyses and IR spectroscopic data of compounds 19 were consistent with the assigned structures. The structures of the 19 were also ascertained by the ${ }^{1} \mathrm{H}$ NMR, ${ }^{13} \mathrm{C}$ NMR and MS measurements (Experimental).

\section{CONCLUSIONS}

The reaction of hydrazonoyl halides 1a-k with thiocarboxanilide derivatives 4 and 14 gave the corresponding 1,3,4-thiadiazole derivatives 7 and 15 similar to the products previously obtained with different thiocarboxanilide derivatives [27]. The reaction of active $\alpha$-chloromethylene ketones with thiocarboxanilide derivatives gave the thiazoline derivatives 19 , contrary to the previous finding we have reported [28] which gave 1,3-oxathiol derivatives.

\section{REFERENCES}

[1] Kormis, G. (1984) 1,3,4-thiadiazoles. In: A. R. Katritzky, Ed., Comprehensive Heterocyclic Chemistry, Pergamon Press, 6, 545-578. 
[2] Hussein, M.A., Kafafy, A.H.N., Abdel-Moty, S.G. and Abou-Ghadir, O.M.F. (2009) Synthesis and biological activities of new substituted thiazoline-quinoline derivatives. Acta Pharmaceutica, 59, 365-382. doi:10.2478/v10007-009-0033-8

[3] Pintilie, O., Profire, L., Sunel, V., Popa, M. and Pui, A. (2007) Synthesis and antimicrobial activity of some new 1,3,4-thiadiazole and 1,2,4-triazole compounds having a D,L-methionine moiety. Molecules, 12, 103-113. doi:10.3390/12010103

[4] Faroumadi, A., Mirzaei, M. and Shafiee, A. (2001) Antitubercular agents, I: Synthesis and antituberculosis activity of 2-aryl-1,3,4-thiadiazole derivatives. Pharmazie, 56, 610-612.

[5] Mamolo, M.G., Falagiani, V., Zanpier, D., Vio, L. and Banfi, F. (2001) Synthesis and antimycobacterial activity of [5- (pyridin-2-yl)-1,3,4-thiadiazol-2-ylthio]acetic acid arylidene- hydrazide derivatives. II Farmaco, 56, 587592. doi:10.1016/S0014-827X(01)01097-7

[6] Zou, X.J., Lai, L.H., Jin, G.Y. and Zhang, Z.X. (2002) Synthesis, fungicidal activity, and 3D-QSAR of pyridazinone-substituted 1,3,4-oxadiazoles and 1,3,4-thiadiazoles. Journal of Agricultural and Food Chemistry, 50, 3757-3760. doi:10.1021/if0201677

[7] Chen, H., Li, Z. and Han, Y. (2000) Synthesis and fungicidal activity against rhizoctonia solani of 2-alkyl (alkylthio)-5-pyrazolyl-1,3,4-oxadiazoles (thiadiazoles). Journal of Agricultural and Food Chemistry, 48, 5312-5315. doi:10.1021/jf991065s

[8] Clerici, F., Pocar, D., Guido, M., Loche, A., Perlini, V. and Brufani, M. (2001) Synthesis of 2-amino-5-sulfanyl1,3,4-thiadiazole derivatives and evaluation of their antidepressant and anxiolytic activity. Journal of Medicinal Chemistry, 44, pp. 931-936. doi:10.1021/im001027w

[9] Palaska, E., Sahin, G., Kelincen, P., Durlu, N.T. and Altionax, G. (2002) Synthesis and anti-inflammatory activity of 1-acylthiosemicarbazides, 1,3,4-oxadiazoles, 1,3, 4-thiadiazoles and 1,2,4-triazole-3-thiones. II Farmaco, 57, 101-107. doi:10.1016/S0014-827X(01)01176-4

[10] Shenone, S., Bruno, O., Ranise, A., Bondavalli, W., Falcone, G., Giordano, L. and Vitelli, M. (2001) 3-Arylsulphonyl-5-arylamino-1,3,4-thiadiazol-2 $(3 \mathrm{H})$ ones as antiinflammatory and analgesic agents. Bioorganic \& $\mathrm{Me}$ dicinal Chemistry, 9, 2149-2153. doi:10.1016/S0968-0896(01)00121-3

[11] Onko., T. Cakir, B. and Sahin, M.F. (2004) Synthesis and antinociceptive activity of 2-[(2-Oxobenzothiazolin-3-yl) methyl]-5-aminoalkyl/aryl-1,3,4-thiadiazole. Turkish Journal of Chemistry, 28, 461-468.

[12] Azab, M.E., El-Hag Ali, G.A.M. and Abd El-Wahab, A.H.F. (2003) Studies on thiazolopyridines: A novel synthesis of bisthiazolopyridines as promising antimicrobial agents. Acta Pharmaceutica, 53, 213-221.

[13] Sondhi, S.M., Singh, N., Lahoti, A.M., Bajaj, K., Kumar, A., Lozach, O. and Meijer, L. (2005) Synthesis of acridinyl-thiazolino derivatives and their evaluation for antinflammatory, analgesic and kinase inhibition activities. Bioorganic \& Medicinal Chemistry, 13, 4291-4299. doi:10.1016/j.bmc.2005.04.017

[14] Mahler, G., Serra, G., Dematteis, S., Saldana, J., Dominguez, L. and Manta, E. (2006) Synthesis and bio- logical evaluation of simplified mycothiazole analogues. Bioorganic \& Medicinal Chemistry Letters, 16, 13091311. doi:10.1016/j.bmcl.2005.11.072

[15] Shih, M. and Ke, F. (2004) Syntheses and evaluation of antioxidant activity of sydnonyl substituted thiazolidinone and thiazoline derivatives. Bioorganic \& Medicinal Chemistry Letters, 12, 4633-4643.

doi:10.1016/j.bmc.2004.06.033

[16] Godefroi, E.F. and Platje, J.Th. (1972) DL-1-(.alpha.-Methylbenzyl)-2-methylimidazole-5-carboxylate esters. Synthesis and pharmacological properties. Journal of $\mathrm{Me}$ dicinal Chemistry, 15, 336-337. doi:10.1021/jm00273a035

[17] Harfenist, M., Soroko, F.E. and Mckenzie, G.M. (1978) 2-(Alkoxyaryl)-2-imidazoline monoamine oxidase inhibitors with antidepressant activity. Journal of Medicinal Chemistry, 21, 405-409. doi:10.1021/jm00202a021

[18] Kornicka, A., Hudson, A.L. and Bednarski, P.J. (2009) Synthesis and biological activity of some 2-imidazolinylhydrazone derivatives. Acta Poloniae Pharmaceutica-Drug Research, 66, 523-534.

[19] Desai, N.C., Bhavsar, A.M. and Baldaniya, B.B. (2009) Synthesis and antimicrobial activity of 5-imidazolinone derivatives. Journal of Postgraduate Medicine, 71, 9094.

[20] Solankee, A., Kapadia, K., Thakor, I., Patel, J. and Lad, S. (2004) Synthesis and Antimicrobial Activity of 1-(4' -Trifluoro methylphenyl and- 2-phenyl-4-(benzylidene/ substituted Benzylidene/2' furylidene/2'-thienylidene)imidazolin-5-ones. Asian Journal of Chemistry, 16, 917920.

[21] Mor, M., Bordi, F., Silva, C., Rivara, S., Zuliani, V., Vacodio, F., Morini, G., Barocelli, E., Ballabeni, V., Impicciatore, M. and Plazzi, P.V. (2000) Synthesis and biological assays of new H3-antagonists with imidazole and imidazoline polar groups. II Farmaco, 55, 27-34. doi:10.1016/S0014-827X(99)00115-9

[22] Giorgioni, G., Claudi, F., Ruggieri, S., Ricciutelli, M., Palmieri, G.F., Di-Stefano, A., Sozio, P., Cerasa, L.S., Chiavaroli, A., Ferrante, C., Orlando and G., Glennon, (2010) Design, synthesis, and preliminary pharmacological evaluation of new imidazolinones as 1-DOPA prodrugs. Bioorganic \& Medicinal Chemistry Letters, 18, 1834-1843. doi:10.1016/j.bmc.2010.01.041

[23] Abunada, N.M., Hassaneen, H.M., Kandile, N.G. and Miqdad, O.A. (2008) Synthesis and biological activity of some new pyrazoline and pyrrolo[3,4-c]pyrazole-4,6dione derivatives: reaction of nitrilimines with some dipolarophiles. Molecules, 13, 1011-1024. doi:10.3390/molecules 13041011

[24] Abunada, N.M., Hassaneen, H.M., Kandile, N.G. and Miqdad, O.A. (2008) Synthesis and antimicrobial activity of some new pyrazole, fused pyrazolo[3,4-d]-pyrimidine and pyrazolo[4,3-e][1,2,4]-triazolo[1,5-c]pyrimidine derivatives. Molecules, 13, 1501-1517. doi:10.3390/molecules 13071501

[25] Abunada, N.M., Hassaneen, H.M., Abu Samaha, A.M. and Miqdad, O.A. (2009) Synthesis and antimicrobial evaluation of some new pyrazole, pyrazoline and chromeno [3,4-c]pyrazole derivatives. Journal of Brazilian Chemical Society, 20, 975-987. 
doi:10.1590/S0103-50532009000500024

[26] Hassaneen, H.M., Shawali, A.S., Khalil, M.S. and Abdallah, T.A.A. (1993) One step synthesis of benzimidazo[2,1-c] $[1,2,4]$ triazole derivatives using hydrazonoyl halides. Heterocycles, 36, 1775-1781. doi:10.3987/COM-93-6344

[27] Hassaneen, H.M., Abbas, I.M., Abdelhadi, H.A., Abdallah, T.A. and Algharib, M.S. (1994) Reactions of 5-oxo2-pyrazolin-4-thiocarboxanilides and 5-oxo-2-oxazolin4-thiocarboxanilides with hydrazonoyl halides. Sulfur Letters, 17, 295-307.

[28] Hassaneen, H.M., Elwan, N.M., Abdelhadi, H.A. and Abdallah, T.A. (1995) Reactions of thioanilides with active chloromethylene compounds. Synthesis of 1,3oxathiol-2-ylidene derivatives. Sulfur Letters, 18, 121128.

[29] Hassaneen, H.M., Harhash, A.H.E., Abunada, N.M., Abdallah, T.A. and Algharib, M.S. (1993) Cyano-(1-methylbenzimidazol-2-yl)thioacetanilide in the synthesis of 2,3-dihydro-1,3,4-thiadiazole derivatives. The Journal of Chemical Research, 194-195.

[30] Argyropoulou, E.C. and Thessalonikeos, E. (1990) Reactions of nitrile oxides and nitrile imines with 4-arylidene2-phenyl-5(4H)-thiazolones. Justus Liebigs Annalen der Chemie, 1097-1100. doi:10.1002/jlac.1990199001198

[31] Johnson, T.B. and Hadley, S.E. (1915) Researches on hydantoins. XXIX. Geometrical isomerism in the hydantoin series. Journal of American Chemical Society, 37, 171-177. doi:10.1021/ja02270a017

[32] Wolkoff, P. (1975) A new method of preparing hydrazonyl halides. Canadian Journal of Chemistry, 53, 13331335. doi:10.1139/v75-183

[33] Hassaneen, H.M., Elwan, N.M., Harhash, A. and Shawali, A.S. (1984) The regioselectivity in the formation of pyrazolines and pyrazoles from nitrile imines. Journal of Heterocyclic Chemistry, 21, 1013-1016. doi:10.1002/jhet.5570210417

[34] Hassaneen, H.M., Mousa, H.A.H., Abed, N.M. and Shawali, A.S. (1988) Chemistry of C-Heteroarylhydrazidoyl halides. Synthesis and reactions of $\mathrm{n}$-(p-nitrophenyl)-c(2-thienyl)-formohydrazidoyl halides. Heterocycles, 27, 695-706. doi:10.3987/COM-87-4381

[35] Shawali, A.S., Hassaneen, H.M. and Ibrahim, H.A. (1990) Synthesis and cycloaddition reactions of N-aryl-2-furohydrazonoyl chlorides. Archives of Pharmacal Research, 13, 126-131.

\section{doi:10.1007/BF02857788}

[36] Aylward, J.B. and Scott, F.L. (1969) Preparation and sovolysis of N1-arylbenzohydrazonyl bromides. Journal of Chemical Society (B), 1969, 1080-1084. doi:10.1039/j29690001080

[37] Eweiss, N.F. and Osman, A. (1980) Synthesis of heterocycles. Part II. New routes to acetylthiadiazolines and alkylazothiazoles. Journal of Heterocyclic Chemistry, 17, 1713-1717. doi:10.1002/jhet.5570170814

[38] Shawali, A.S., Eweiss, N.F., Hassaneen, H.M. and Algharib, M.S. (1975) Synthesis and rearrangement of ethyl aryloxyglyoxalate arylhydrazones. Bulletin of the Chemical Society of Japan, 48, 365-366. doi: $10.1246 /$ bcsj. 48.365

[39] Shawali, A.S. and Osman, A. (1971) Synthesis and reactions of phenylcarbamoylarylhydrazidic chlorides. Tetrahedron, 27, 2517-2528. doi:10.1016/S0040-4020(01)90753-7

[40] Shawali, A.S. and Abdelhamid, A.O. (1976) Reaction of dimethylphenacylsulfonium bromide with N-nitrosoacetarylamides and reactions of the products with nucleophiles. Bulletin of the Chemical Society of Japan, 49, 321-324. doi:10.1246/bcsj.49.321

[41] Farag, A.M. and Algharib, M.S. (1988) Synthesis and reactions of C-(2-thenoyl)-N-arylformahydrazidoyl bromides. Organic Preparations and Procedures International, 20, 521-526. doi:10.1080/00304948809356298

[42] Hassaneen, H.M., Shawali, A.S., Elwan, N.M. and Abunada, N.M. (1992) Reaction of 1-(2-naphthoyl) methyl-2-dimethylsulfonium bromide with N-nitroso-Narylacetamides and reactions of the products with some nucleophiles. Sulfur Letters, 13, 273-285.

[43] Huisgen, R., Grashey, R., Seidel, M., Knupfer, H. and Schmidt, R. (1962) 1,3-Dipolare Additionen, III. Umsetzungen des Diphenylnitrilimins mit Carbonyl und Thiocarbonyl-Verbindungen. Justus Liebigs Annalen der Chemie, 658, 169-180. doi:10.1002/jlac.19626580115

[44] Abdelhamid, A.O. and Abdou, S.E. (1987) Reactions of cyanothioacetamide with hydrazidoyl halides: A novel synthesis of some hydrazidoyl sulfides and triazole derivatives. Sulfur Letters, 6, 41-47.

[45] Mohareb, R.M., Sherif, S.M., Abdel-Aal, F.A.M. and Sayed, N.I.A. (1990) One-pot synthesis of polyfunctionally substituted 2,3-dihydrothiazoles and thiazolidinones. Justus Liebigs Annalen der Chemie, 1143-1146. 